\title{
Bronchial Cast in a Child with Hemoptysis
}

\author{
Ju Hyun Jin ${ }^{1}$, In Suk Sol${ }^{2}$, Mi-Jung Lee ${ }^{3}$, Hyo Sup Shim ${ }^{4}$, Joo Han Song ${ }^{5}$, Nam Kyun Kim ${ }^{6}$, Kyung Won Kim² and Myung Hyun Sohn² \\ ${ }^{1}$ Department of Pediatrics, National Health Insurance System Ilsan Hospital, Goyang, Korea \\ ${ }^{2}$ Department of Pediatrics, Severance Hospital, Institute of Allergy, Yonsei University College of Medicine, Seoul, Korea \\ ${ }^{3}$ Department of Radiology, Severance Hospital, Yonsei University College of Medicine, Seoul, Korea \\ ${ }^{4}$ Department of Pathology, Severance Hospital, Yonsei University College of Medicine, Seoul, Korea \\ ${ }^{5}$ Division of Pulmonary and Critical Care Medicine, Department of Internal Medicine, Severance Hospital, Institute of Chest Disease Yonsei University College of \\ Medicine, Seoul, Korea \\ ${ }^{6}$ Division of Pediatric Cardiology, Department of Pediatrics, Congenital Heart Disease Center, Severance Cardiovascular Hospital, Yonsei University College of Medicine, \\ Seoul, Korea
}

\section{Abstract}

Bronchial cast is a rare clinical problem that is more often reported in children than in adults. Reported mean ages ranges from 4 to 12.8 years. It is associated with underlying diseases such as asthma, cystic fibrosis, allergic bronchopulmonary aspergillosis, and congenital heart defects. Bronchial casts vary in size, and clinical presentations may differ depending on the size of the casts. Prognosis also depends on the associated disease and the type of cast, ranging from rapid recovery after cast removal to life-threating respiratory impairment. It is generally expectorated spontaneously or with a cough, but sometimes needed bronchoscopy to remove from bronchi. Moreover, the understanding of pathogenesis is still insufficient. There have been reported a few cases of bronchial cast with hemoptysis in patients without an underlying disease. Herein, we report a patient with bronchial cast following hemoptysis, secondary to an aortopulmonary collateral artery, in the absence of a lung or systemic disease.
\end{abstract}

Keywords: Bronchial cast; Hemoptysis; Aortopulmonary collateral artery; Pulmonary hemorrhage

\section{Introduction}

Bronchial casts are characterized by a "pudding-like" obstructive plug conforming to the anatomy of the involved bronchi $[1,2]$. The clinical or radiologic findings of bronchial casts are variable according to its sizes or site. In addition, they are usually associated with various diseases such as asthma, cystic fibrosis, allergic bronchopulmonary aspergillosis, acute chest syndrome in sickle cell disease, and congenital heart defects [3].

Several classifications based on underlying disease, pathology and morphology have been suggested to explain the bronchial cast [2-4]. However, these classifications are not appropriate in all cases. There have been a few reports on bronchial cast in children without underlying diseases $[3,5,6]$. We report the case of a 12 -year-old girl who expectorated a bronchial cast following hemoptysis, secondary to an aortopulmonary collateral artery (APC), in the absence of a lung or systemic disease.

\section{Case Report}

A 12-year old girl was admitted because of hemoptysis $(\sim 100 \mathrm{~mL}$ blood) for 2 days. She had no relevant medical history besides Hashimoto thyroiditis without taking any medications. She had no history of any pulmonary or cardiac disorder. Although she experienced the same episode two months before, hemoptysis was resolved spontaneously without any management. She had mild cough and rhinorrhea but unremarkable. She had no any other respiratory symptoms.

On physical examination at this admission, chest auscultation revealed good bilateral breath sounds without wheezing or rale, and no heart murmurs were detected. There were no other abnormalities such as digital clubbing. Her hemoglobin and hematocrit were $11.9 \mathrm{~g} / \mathrm{dL}$ and $38 \%$, respectively. Arterial blood gas analysis and coagulation test were normal. The tuberculosis tests were all negative. Antinuclear antibody, anti-DNA, rheumatic factor, and antinuclear cytoplasmic antibody tests were negative for vasculitis. Her thyroid hormone levels were within the normal range. Chest radiography showed ground glass opacity (GGO) and consolidation on the right lung. There was no bleeding focus on flexible laryngoscopy, esophagogastroduodenoscopy, and paranasal sinus computed tomography (CT). Chest CT showed bronchial wall thickening in the right upper lung with surrounding patchy GGOs and centrilobular nodules (Figure 1A). An enlarged main pulmonary artery was detected. Although slight hypertrophy of branches of the right internal mammary artery was observed on neck CT, there was no clear evidence of active bleeding (Figure 1B).

On the third hospital day, the patient expectorated a reddish pudding-like cast (Figure 2). She underwent a bronchoscopy, revealing active endobronchial bleeding from the right upper lobe apical segment (Figure 3). She underwent cardiac catheterization for detection and embolization of bleeding vessels. An APC from the internal mammary artery to the right upper lung was detected on catheterization (Figure 4). Embolization was performed with four $2 \times 10 \mathrm{~mm}$ MReye embolization coils (Cook Medical Inc., Bloomington, IN, USA). There were no pulmonary hypertension and cardiac anomalies. After the embolization, the patient was stable and did not exhibit hemoptysis. Follow-up chest CT performed 3 days after embolization showed that the patchy peribronchial GGOs decreased. The patient was discharged and had no recurrent hemoptysis during the 6-month follow-up. The pathology of the cast was inflammatory fibrinous exudate with blood clot.

*Corresponding author: Kyung Won Kim, Department of Pediatrics, Severance Children's Hospital, Yonsei University College of Medicine, 50 Yonsei-ro, Seodaemun-gu, Seoul, 03722, Korea, Tel: 82222282050; Fax: 8223939118, E-mail: kwkim@yuhs.ac

Received April 12, 2017; Accepted October 16, 2017; Published October 23, 2017

Citation: Jin JH, Sol IS, Lee MJ, Shim HS, Song JH, et al. (2017) Bronchial Cast in a Child with Hemoptysis. J Pulm Respir Med 7: 425. doi: 10.4172/2161 105X.1000425

Copyright: (c) $2017 \mathrm{Jin} \mathrm{JH}$, et al. This is an open-access article distributed under the terms of the Creative Commons Attribution License, which permits unrestricted use, distribution, and reproduction in any medium, provided the original author and source are credited. 
Citation: Jin JH, Sol IS, Lee MJ, Shim HS, Song JH, et al. (2017) Bronchial Cast in a Child with Hemoptysis. J Pulm Respir Med 7: 425. doi: $10.4172 / 2161-105 X .1000425$

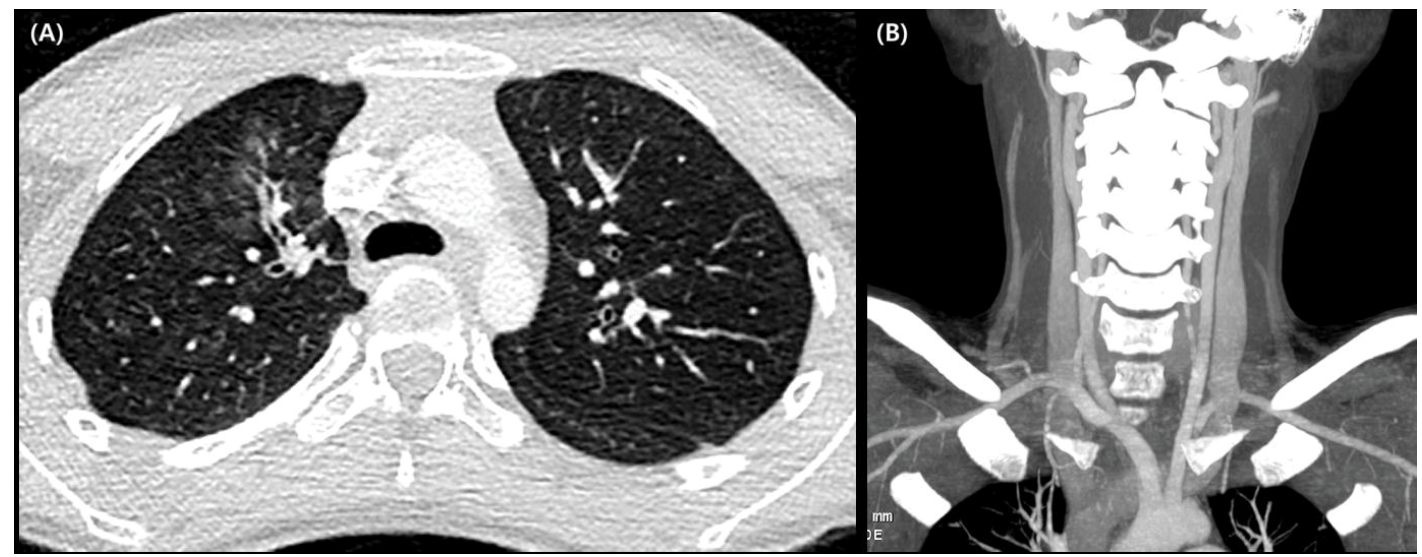

Figure 1: (A) Chest computed tomography (CT) scan showing bronchial wall thickening in the right upper lung with surrounding patchy ground glass opacities and centrilobular nodules. There is no definite endobronchial lesion or atelectasis. (B) Neck CT scan showing a hypertrophied branch (arrow) of the right internal mammary artery.
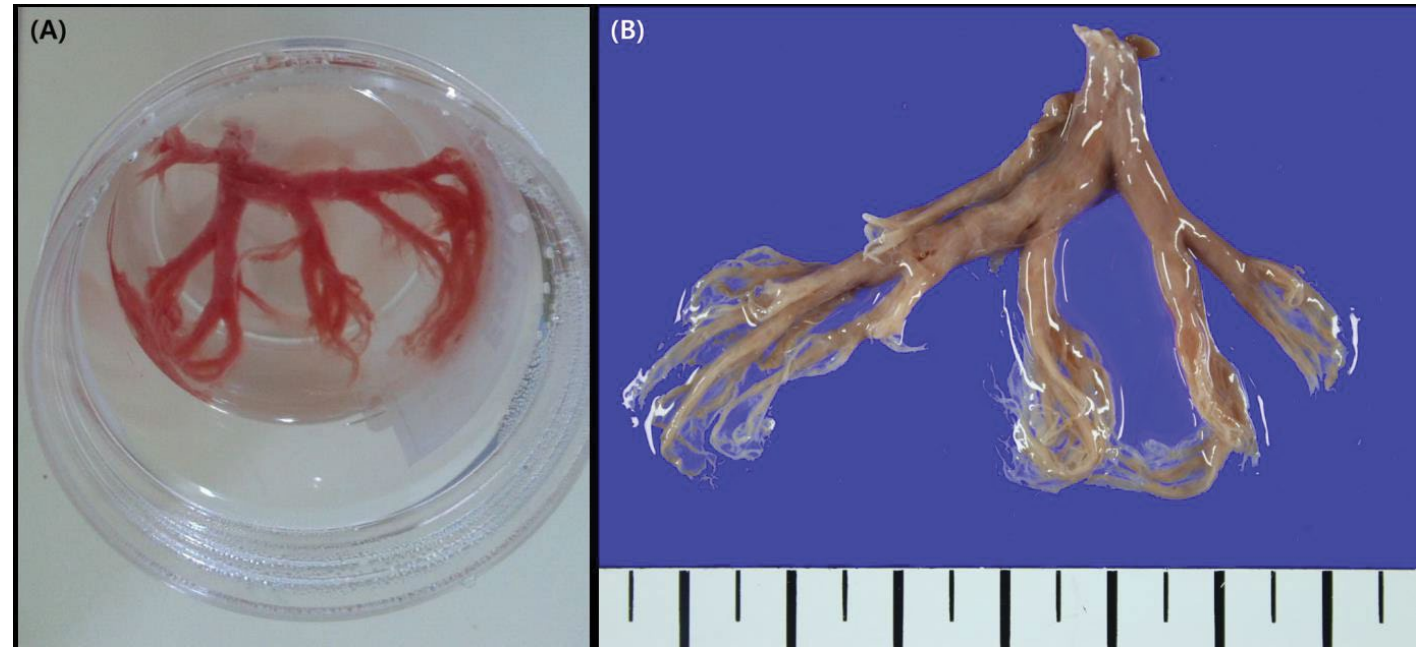

Figure 2: (A) Bronchial cast spontaneously expectorated by the patient. (B) Bronchial cast after fixation: preserved anatomy of the bronchial tree and the pathology of inflammatory fibrinous exudates with blood clot.

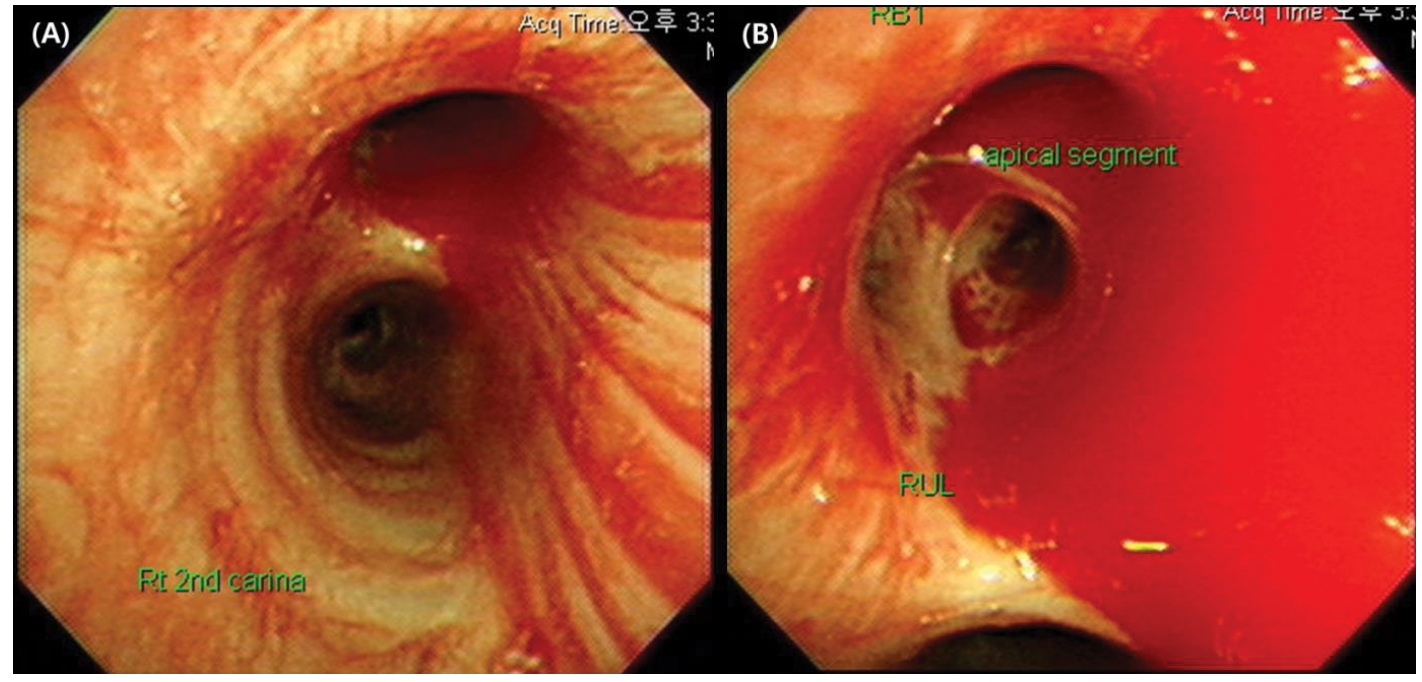

Figure 3: Bronchoscopy images obtained at (A) the right second carina and (B) the right apical segment showing active endobronchial bleeding from the apical segment of the right upper lobe. 

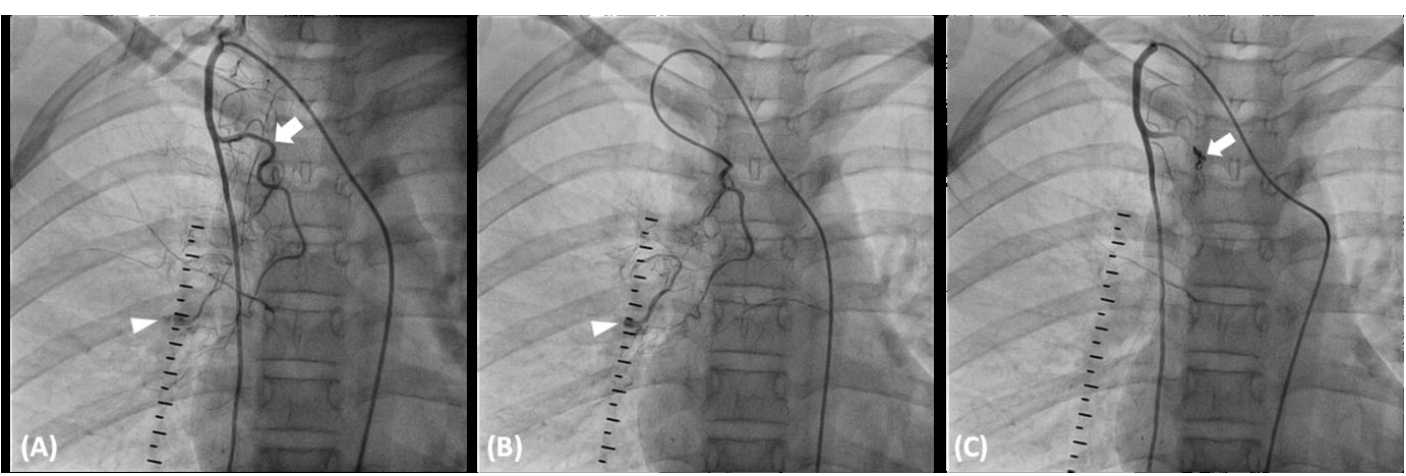

Figure 4: Serial angiogram images. (A) Angiogram of the right internal mammary artery. The collateral artery (arrowhead) drains from the branch of the right internal mammary artery (arrow) to the right middle lung field. (B) Selective angiogram at the branch of the right internal mammary artery. The collateral artery (arrow) that drains to the right bronchus is observed more clearly. (C) Final angiogram of the right internal mammary artery after coil embolization. Coil (arrow) is observed. The abnormal vessel that drains to the right bronchus is no longer observed.

\section{Discussion}

Bronchial cast is an unusual condition that can be a life-threatening emergency if mechanical obstruction of major airways occurs [6]. Although bronchial cast can occur at all ages, many of the reported cases were in children, especially as a complication of corrective surgery for congenital heart defects [7]. The true prevalence of bronchial cast is unknown, because it is difficult to differentiate bronchial cast from mucus plug in children with asthma [2]. However, there are a few reports on bronchial cast in children without underlying diseases $[3,5,6]$.

The pathogenesis is unclear; however, there have been several attempts to explain the formation of casts. Seear et al. divided bronchial casts into type I and type II [3]. Type I (inflammatory) casts consist of fibrin with dense eosinophilic inflammatory infiltrates, and are associated with primary pulmonary disease and bronchial inflammation. Type II (acellular) casts consist of mucin with hypocellular infiltrates, and are associated with cyanotic heart disease [3]. Concerning these classifications, the suggested etiologies include abnormal lymphatic drainage, increased mucus secretion due to elevated pulmonary venous pressure, low cardiac output, or inflammatory response $[8,9]$.

APCs are also usually associated with congenital cardiac disease [10]. In our case, there was an APC without cardiac anomalies. The cast consisted of inflammatory fibrinous exudates on pathological examination. Lung hyperperfusion can be induced by systemic arterial blood flow through aberrant arteries like APCs, and can cause damage in the alveolar compartment $[11,12]$. The exact pathophysiology is unknown; however, it is presumed that bronchial inflammation is caused by APCs.

Clinical and radiologic findings vary with the size of cast, and associated illness [3]. Patients often experience dyspnea, wheezing, pleuritic chest pain, and fever. Physical examination may reveal decreased breath sound or wheezing. Our patient had recurrent hemoptysis; however, hemoptysis is not a usual symptom of bronchial cast [13]. Chest radiography often demonstrates atelectasis of the involved area or hyperinflation of the contralateral side. The chest radiographic findings of our patient (GGO and consolidation without endobronchial lesions due to aspiration of blood) were also not typical features. The diagnosis is usually confirmed with bronchoscopy demonstrating airway obstruction with a thick cast or by expectoration of the cast [2]. In our patient, bronchial cast was diagnosed after its expectoration. Assessment for underlying diseases should be done simultaneously. Our patient also underwent various tests; however, there were no definite underlying diseases except for an APC.

The treatment of bronchial casts includes acute cast removal and therapy of underlying diseases to prevent cast recurrence [8]. Cast removal through bronchoscopy or expectoration is considered the standard therapy, and medical treatments such as mucolytics, bronchodilators, inhaled and oral corticosteroids, and antibiotics are used if necessary. In this case, the bronchial cast was removed spontaneously through expectoration, and embolization of the APC, which was considered the cause of the bronchial cast, was performed.

In summary, we report the case of a patient with bronchial cast due to APC in the absence of a cardiopulmonary disease. On the basis of the cast pathology, we suspected that bronchial inflammation induced by the APC resulted in bronchial cast formation.

\section{Acknowledgement}

This work was supported by the Korea Research Foundation Grant funded by the Korean Government (KRF-2015-31-0618).

\section{References}

1. Fairshter RD, Riley CA, Hewlett RI (1979) Large bronchial casts. Arch Intern Med 139:522-525

2. Madsen P, Shah SA, Rubin BK (2005) Plastic bronchitis: New insights and a classification scheme. Paediatric Resp Rev 6: 292-300.

3. Seear M, Hui H, Magee F, Bohn D, Cutz E (1997) Bronchial casts in children: A proposed classification based on nine cases and a review of the literature. American journal of respiratory and critical care medicine 155: 364-370.

4. Beitmann M (1902) Report of a case of fibrinous bronchitis, with a review of all cases in the literature. Am J Med Sci 123: 304-329.

5. Kim YH, Choi HJ, Kim JO, Hyun MC (2009) Plastic bronchitis in children: 2 cases. Korean J Pediatr 52: 832-836.

6. Eberlein $\mathrm{MH}$, Drummond MB, Haponik EF (2008) Plastic bronchitis: A management challenge. Am J Med Sci 335: 163-169.

7. Turgut T, Ozercan IH, Kaplan M (2014) A case of plastic bronchitis. Archives of Iranian Medicine 17: 589-590.

8. Kunder R, Kunder C, Sun HY, Berry G, Messner A, et al (2013) Pediatric plastic bronchitis: Case report and retrospective comparative analysis of epidemiology and pathology.

9. Schumacher KR, Singh TP, Kuebler J, Aprile K, O'Brien M, et al. (2014) Risk factors and outcome of fontan-associated plastic bronchitis: A case-control study. J American Heart Asso 3: e000865.

10. Frank F, Laskari C, Bierman FZ (1996) Additional aortopulmonary collaterals 
Citation: Jin JH, Sol IS, Lee MJ, Shim HS, Song JH, et al. (2017) Bronchial Cast in a Child with Hemoptysis. J Pulm Respir Med 7: 425. doi: 10.4172/2161-105X.1000425

in patients referred for coil occlusion of a patent ductus arteriosus. Cathet Cardiovasc Diagn 37: 5-8.

11. Pelizzo G, Calcaterra V, Mannarino S, Moramarco LP, Leati G, et al. (2015) Aortopulmonary collateral artery in prenatal exposure to carbamazepineendovascular therapy and technical considerations: A case report. Journal of medical case reports 9: 183.
12. Kozian A, Schilling T, Fredn F, Maripuu E, Rcken C, et al. (2008) One-lung ventilation induces hyperperfusion and alveolar damage in the ventilated lung: An experimental study. Br J Anaesth 100: 549-559.

13. Wang G, Wang L, Jiang L, Wang L, Mao B (2006) Effective use of corticosteroids in treatment of plastic bronchitis with hemoptysis in chinese adults. Acta Pharmacol Sin 27: 1206-1212. 